\title{
Efectos del aprendizaje basado en problemas en la competencia instrumental traductora
}

\section{Effects of problem-based learning on instrumental translation competence}

\author{
Daniel Silva-Nieves, ${ }^{1 a}$ Doris Fuster-Guillén, ${ }^{2}$ Roger Pedro Norabuena-Figueroa, ${ }^{3}$ \\ Edwin Hernán Ramírez-Asís ${ }^{4}$ y Ana Beatriz Aguirre-Jiménez ${ }^{5}$ \\ Universidad César Vallejo, Lima, Perú ${ }^{15}$ \\ Universidad Nacional Mayor de San Marcos, Lima, Perú ${ }^{2}$ \\ Universidad Nacional Santiago Antúnez de Mayolo, Huaraz, Perú ${ }^{34}$
}

(iD) Orcid ID: https://orcid.org/0000-0002-9897-9805 ${ }^{1}$

(iD) Orcid ID: https://orcid.org/0000-0002-7889-22432

(iD) Orcid ID: https://orcid.org/0000-0003-3731-98433

iD Orcid ID: https://orcid.org/0000-0002-9918-7607

iD Orcid ID: https://orcid.org/0000-0002-6693-133X

Recepción: 10 de octubre de 2019

Aceptación: 08 de febrero de 2020

\begin{abstract}
Resumen
El presente estudio se propuso establecer los efectos del aprendizaje basado en problemas en la competencia instrumental traductora en estudiantes de la escuela de traducción e interpretación de una universidad privada de la ciudad de Lima. Asimismo, se concentra en la búsqueda de explicaciones respecto a la habilidad en el saber traducir, habilidad presente y ausente en estudiantes universitarios, y si dicha competencia se debía a las múltiples tareas que se asignaban. Esta investigación se desarrolla dentro del paradigma neopositivista, enfoque cuantitativo, tipo sustantivo, diseño no experimental, y método hipotético deductivo. La población estuvo constituida por 233 estudiantes y la muestra por 145 , calculada por muestreo probabilístico aleatorio, a los cuales se les suministró una batería de preguntas (escala tipo Likert), las cuales fueron validadas por contenido, la fiabilidad de consistencia alta para ambas variables establecida por Alpha de Cronbach. La contrastación de hipótesis se realizó a través del coeficiente de correlación Rho de Spearman cuyo valor fue de .852 ( $\mathrm{p}>.05)$, del cual se concluyó que el aprendizaje basado en problemas no influye significativamente en la competencia instrumental traductora de los estudiantes; sin embargo, se identificó que el $80 \%$ de
\end{abstract}

${ }^{\mathrm{a} C}$ Correspondencia al autor: daniel.idiomas.007@gmail.com 
estudiantes presentan estrategias en la elaboración de tareas, así como una competencia instrumental traductora.

Palabras clave: Traducción e interpretación, aprendizaje basado en problemas, competencia instrumental traductora.

\begin{abstract}
This study sets out to establish the effects of problem-based learning on translator instrumental competence in students of the School of Translation and Interpretation of a private university in the city of Lima. Focused on the search for explanations regarding ability to know how to translate, present and absent in university students and if this competence was due to the multiple tasks assigned. Developed within the neo-positivist paradigm, quantitative approach, substantive type, non-experimental design, deductive hypothetical method. The population consisted of 233 students and the sample of 145 calculated by random probabilistic sampling, which were supplied with a battery of questions (Likert scale). Which were validated by content, the reliability of high consistency for both variables established by Cronbach's Alpha. The hypothesis test was carried out through Spearman's Rho correlation coefficient, whose value was 0.852 (p> 0.05), from which it was concluded that problem-based learning does not significantly influence the translational instrumental competence of students, however We identify that $80 \%$ of students present strategies in the elaboration of tasks as well as instrumental translation competence.
\end{abstract}

Keywords: Translation and interpretation, problem-based learning, translator instrumental competence.

\title{
Introducción
}

En los recientes estudios y cursos de enseñanza de idiomas extranjeros se puede observar importantes cambios en el abordaje del aprendizaje de lenguas modernas. Estos cambios implican reflexiones teóricas, investigativas, y sobre todo, pedagógicas. Es por ello que, desde las aulas, se viene generando una permanente innovación que critica al modelo hegemónico; esto lleva a la necesidad de una modelación alterna para la enseñanza-aprendizaje de un idioma extranjero. Los años de experiencia subrayan la idea de que no existiría una metodología perfecta e indubitable que logre sin dificultad el aprendizaje de una lengua. Es precisamente por ello que se debe propugnar una constante mejora, revisión y, sobre todo, experimentación de métodos, estrategias y demás que permitan no solo enriquecer sino cuestionar y mejorar la enseñanzaaprendizaje de idiomas, ya que cada lengua tiene su peculiar idiosincrasia idiomática (Marangon, 2012). El ejercicio de poner en práctica lo novedoso, los aportes en el desarrollo de actividades, las lecturas, la expresión oral y escrita en general, lo pragmático e incluso, desde formas alternativas de comunicación que impliquen textos 
continuos o discontinuos, infografías y organizadores visuales, serán una contribución importante para el avance en las investigaciones (Perkins, 2015). Por ejemplo, el uso de mapas mentales y otros organizadores para la comprensión y la producción de textos están tomando con mayor pronunciamiento una interesante propuesta de ayuda para los docentes tanto en lenguas maternas (Novoa 2012, 2018, 2019; Nuñez y Novoa et al, 2018) como en segundas lenguas. En este sentido, las unidades didácticas comienzan con unas pruebas tipo diagnóstico y finalizan con ensayos tipo autoevaluación. En estas últimas, se identifica las evaluaciones basadas en tareas, interesante estrategia que en la metodología de la enseñanza de lenguas permite emplearlas como herramientas. Es en este contexto que las tareas se transforman en una competencia instrumental efectiva en la traducción, pues permiten plantear una tarea específica comunicacional, y se logra no solo una traducción sino una interpretación idónea de una lengua hacia otra.

Por otro lado, la enseñanza-aprendizaje basado en problemas ha sido empleada con mucho éxito en países como Cuba, donde-mediante diversas disciplinas lingüísticas como la hermenéutica y la pragmática - se puede lograr una enseñanza no solo más fluida sino más eficaz de un idioma (Jerez y Garófalo, 2012). Cabe resaltar el énfasis en la competencia instrumental traductora, considerada en Europa y algunos países de Latinoamérica como un componente imprescindible de la competencia traductora. Esta implica no solo un conocimiento teórico y gramatical de la lengua, sino la necesidad de emplear el conocimiento idiomático como instrumento comunicacional basado en la satisfacción real y concreta en un acto comunicativo (Wang, 2016; Escarrá, 2013; Massey y Ehrensberger-Dow, 2011). En otras investigaciones, se la denomina como competencia lingüística (Mizab y Bahloul, 2016) con la misma connotación, pero otorgándole la misma relevancia en lo pragmático, sobrepasando lo meramente gramatical y convencional. En suma, todos los estudios referenciados entienden que la problemática de la traducción y de la enseñanza de una lengua extranjera, se agiliza cuando se cuenta con una estrategia instrumental que asegura la comunicación real. Este ejercicio es simple y concreto en los hablantes, ya que sirve no solo para una comprensión, sino también para la traducción e interpretación de textos de una lengua hacia otra.

En otro orden, el título de traductor e interpretador ha implicado que se considere a la traducción no como una mera transcripción de una lengua a otra, sino un añadido interpretativo que se basa en el conocimiento pragmático de la idiosincrasia de 
la lengua de origen como de la lengua de destino. Denominada como "traductorado" en algunos países latinoamericanos, requiere para su culminación conocimientos de fonología, lexicología, sintaxis, semántica, fonética $y$, últimamente, de lingüística textual, hermenéutica, pragmática y hasta de teoría de la recepción. Esto motiva que el traductor sea un competente mediador lingüístico, interactuando como un vínculo efectivo y solvente en el ejercicio de dar a entender a alguien o lo que se dice o se ha escrito en una lengua extranjera. Para Galán (2009) la competencia más idónea en un traductor profesional es la sub competencia extralingüística y la sub competencia instrumental (Massey y Ehrensberger-Dow, 2011).

Cabe señalar que la carrera de traducción e interpretación se ha consolidado solo en cuatro universidades en el Perú, centralizadas en la ciudad de Lima. Y aunque aún no se ubica en el currículo de otras universidades, se suelen plantear como una extensión de la carrera de lingüística. Sobre este punto, Praeli (2016) puntualiza que la falta de rigor educativo-y, por ende, de calidad de los traductores-es consecuencia de la exigua disposición de tiempo que se le brinda para su estudio, y que además no se la considera una carrera en sí; a pesar de que cada vez el mercado necesita personas capaces de traducir textos, interpretarlos y analizarlos en otra lengua de origen. Al no haberse consolidado en una mención específica en todas las universidades, las pocas que existen a veces solo se limitan a brindar conocimientos generales de la carrera, y no se especializan, sabiendo aún que existen idiomas tan comerciales e internacionales como el inglés, el chino, el francés y el alemán. En este sentido, Wong (2014) proyecta las competencias de traducción en el empleo de portafolios y rúbricas evaluativas (Lambraño, 2014) como una suerte de operacionalizaciones destinadas a desarrollar, asegurar y consolidar la agilidad traductora e interpretativa de un idioma en particular. Por ello, Mizab y Bahloul (2016) resaltan que la competencia lingüística es probablemente lo más importante en el ejercicio pleno de una lengua, prescindiendo de asignarle mayor interés a otras competencias como las gramaticales, ortográficas y de puntuación. Wang (2016) remarca lo importante que significa el desarrollo de lo instrumental como una competencia en un acto comunicativo real. Para ello, no cierra las puertas a la tecnología; todo lo contrario, amplía el espectro de las herramientas a las TIC, expresadas en diversos aplicativos de traducción en línea que ahora circulan en el ámbito virtual-uno de estos lleva el nombre de Traducción 2.0. A pesar de ello, el empleo de aplicativos y otros programas informáticos todavía es reducido. Torres 
(2015) manifiesta en ellas poco relieve en el rol traductor, ya que virtualmente no asegura, ni mucho menos consolida, la competencia instrumental en el ejercicio traductor.

En todo este amplio panorama, la relación de la competencia traductora y el aprendizaje basado en tareas ha sido poco revisado en nuestro país. En el centro de estudios universitarios donde se ha realizado la presente investigación tampoco se ha encontrado investigaciones sobre el particular. Es por este motivo que los hallazgos de este estudio abrirán nuevas posibilidades para posteriores investigaciones relacionadas con la traducción e interpretación. La relevancia de estudiar esta relación un tanto desatendida en el ámbito de la traducción y la interpretación es el hecho que, con la competencia traductora instrumental más allá de la competencia lingüística, está explicada de acuerdo a los requerimientos actuales, donde el mundo necesita traductores que no solo trasladen con regular mérito un documento hacia otro en cierta lengua, sino que esta competencia debe y tiene que ser ágil y rápida y así dar una respuesta idónea a los usuarios (Silva, 2017).

\section{Aprendizaje basado en problemas}

El aprendizaje basado en problemas (ABP) surge de los vocablos ingleses problem-based learning y se gestó en la Universidad Case Western Reserve, en EEUU, y en la Universidad de McMaster en Canadá (Mora, 2011). Este tipo de aprendizaje se desarrolló dentro de un ámbito de transición de una metodología educativa en idiomas fundamentada en fuertes bases teóricas y conceptuales hacia otra sustentada en una perspectiva procedimental y pragmática, que permitan al hablante no solo expresarse en otro idioma, sino buscar la interpretación cabal del sentido comunicativo a través de la solución de un problema comunicacional puntual. Este tipo de concepción ejerce una relevancia protagónica en la formación de nuevos traductores que entiendan no solo las exigencias de la carrera, sino que se proyecten hacia una empleabilidad que, en esencia, solucione problemas comunicaciones reales en el quehacer diario. En este contexto, Recino y Laufer (2013) consideran esa concepción como una línea metodológica orientada al estudiante, para que éste pueda construir por sí solo un conocimiento pragmático más efectivo por su propia cuenta. El ABP surgió a inicios de la década de los sesenta, aplicándose primera vez en la Universidad McMaster de Ontario (Canadá). El secreto del éxito del ABP es que promueve en el estudiante la auto preparación 
teórica y el ejercicio paulatino e incesante de habilidades prácticas para resolver problemas comunicativos con idoneidad y suficiencia (Jerez y Garófalo, 2012).

Por su parte, Lobato (2011) entiende el ABP como la estrategia pedagógica que se le brinda al usuario para que simule una situación real de comunicación y aplique por necesidad una determinada traducción. En esta, no solo se limitará a la transcripción de un idioma a otro, sino que le inyectará su interpretación (pre-traducción previa) y logrará captar el sentido y la intención del emisor y del mensaje, así como la interacción con el contexto y las circunstancias del acto comunicativo. En este sentido, Lobato resalta la necesidad de plantear una pre-traducción y otra traducción en sí misma. Este proceso se orienta a la construcción de materiales didácticos referidos a un determinado hecho comunicativo, donde el conocimiento previo es determinante en la construcción final de una traducción o interpretación.

Estaire (2011) señala al ABP como una herramienta útil en las tareas de comunicación y tareas de apoyo lingüístico. Además, conceptualiza al ABP como aquello que enfatiza lo práctico como el eje primordial de todo acto comunicativo. Asimismo, menciona que, para culminar con los objetivos planteados, es importante que se diseñen tareas específicas que involucren lo pragmático y real, por ejemplo, lo relevante de los saludos y expresiones fijas. Se aprende más de un idioma al conocerlo y sobre todo si se usa una frase fija de saludo o despedida, que conocer las desinencias del verbo empleado al saludar. Estas formas prácticas de desarrollar hábitos comunicacionales le permitirán al aprendiz incluirse, de manera rápida y significativa, en una reflexión teórica o gramatical.

En otro orden, las tareas de comunicación forman parte de la unidad total y se constituyen como el eje primordial de cualquier unidad didáctica y sesión de aprendizaje. Se orientan para la adquisición de una segunda lengua. Estas tareas pueden ser escritas u orales y permiten que los estudiantes capten en esencia los significados de las expresiones en segunda lengua, así como las intencionalidades del acto comunicativo. Un objetivo a largo plazo es que los alumnos lleguen al nivel de poder pensar en términos de la lengua extranjera (Cerdas y Ramírez, 2015).

De otro lado, las tareas de apoyo lingüístico se basan en criterios de índole lingüístico configurados a partir del idioma que se desea aprender. Para ello, se establecen actividades específicas relacionadas con actos comunicativos y remarcando la corrección idiomática por encima de la fluidez (Balcarcel, 2006). La corrección es importante en tanto que sea una actividad constante y activa de los participantes; y esto 
solo puede ser posible si existe una suerte de negociación en lo formal, o cuando el aprendiz está obligado a pensar y responder luego de su reflexión lingüística al profesor sobre la manera correcta en que debió ser determinada expresión que el docente detectó como incorrecta. La corrección es más funcional cuando hay una retroalimentación por parte del profesor que remarca y valida la corrección o la descarta. En estas actividades, el profesor debe procurar que todas tengan significatividad en sí mismas, de tal forma que se aproveche la situación y el contexto comunicacional. Es por ello que se recomienda que las actividades estén enfocadas en objetivos alcanzables que impliquen que los aprendices además de aprender algo, sean capaces de comunicarse con efectividad y eficiencia. Paralelamente debe darse un procesamiento de tal forma que se dé la interpretación final de todo el acto comunicativo, así como un resultado pragmático de cada tarea planteada (Estaire, 2011).

\section{Competencia instrumental traductora}

Esta se fundamenta, según Torres (2015), en: (a) una didáctica convencional o tradicional — una forma específica de enseñar la traducción bajo el modelo clásico; (b) un estudio de contraste donde, a través del ejercicio comparativo entre la gramática y las características socioculturales de dos lenguas, se pueda generar el aprendizaje; (c) el estilo comparado, donde la estilística también cobra importancia en el sentido de que se pueda expresar formas y modos de escritura, oralidad y otras modalidades de comunicación tanto en lo formal e informal; (d) el aprendizaje entendido por objetivos o competencias comunicativas logradas; (e) la construcción social; (f) la perspectiva de resolución de problemas al traducir; y (g) el desarrollo formativo por competencias específicas de aprendizaje de una lengua.

En este contexto, Kelly (2002) entiende que un traductor eficaz debe ser en conjunto hábil, capaz, diestro, competente y con buena actitud para el aprendizaje de una lengua con su correspondiente ejercicio de traducción e interpretación. Solo ellos son los profesionales, aunque también menciona que existen buenos traductoresllamados traductores nativos o no profesionales - que también realizan buen ejercicio de traducción, pero no cuentan con un bagaje académico que podría completar su buena práctica de traducción e interpretación. Ante esto, Boehm (2013) menciona que los traductores, además de ser hábiles manejando conscientemente la traducción, son personas que tienen un sustento no solo lingüístico, sino social y cultural en cuanto a la lengua foránea que están traduciendo. A estas las denomina "competencias propiamente 
lingüísticas” y a las otras, “extralingüísticas.” Además de estas, los buenos traductores necesitan un empleo efectivo de la lengua extranjera para enfrentar problemas de traducción donde las interferencias - tanto lingüísticas como culturales-oscurezcan la traducción e interpretación. Para ello, el traductor debe ser creativo, culto e intuitivo a la vez, debiendo estar preparado ante cualquier situación. Para lograrlo, Kelly (2002) plantea algunos requisitos primordiales para alcanzar una efectiva competencia instrumental traductora: empleo eficiente de fuentes documentarias, donde se reconozca y califique lo fiable de las fuentes; la búsqueda de terminología idónea de acuerdo a los objetivos y los contextos; y, el uso eficiente de plataformas, aplicaciones y otras TIC relacionadas a la traducción.

\section{Metodología}

El presente modelo de investigación es abordado desde el positivismo y fundamentado en resultados y juicios empíricos para no ser refutados (Meza, 2010). El enfoque elegido fue el cuantitativo, entendido como un enfoque basado en un sustento matemático y estadístico. Asimismo, esta investigación sustantiva se desarrolla en un método hipotético-deductivo, y como diseño se optó por el no experimental. La población de estudio estuvo conformada por 233 estudiantes de la escuela de traducción e interpretación de una universidad privada de Lima. De esta población se tomó una muestra a través de un análisis probabilístico de tipo aleatorio simple. Por ello, el cálculo a través de una fórmula probabilística (Feedback Networks Technologies, 2017) resultó en 145 estudiantes.

La técnica de recojo de información elegida fue la encuesta y se optó como instrumento a un cuestionario para ambas variables. Para el respaldo de este instrumento de ABP se utilizó los componentes propuestos por Estaire (2011), que consta de 20 ítems presentados como afirmaciones; se realizó la validez de contenido a través del juicio de expertos especialistas en estrategias didácticas y se realizó la fiabilidad de los instrumentos a través del Alfa de Cronbach, cuyos valores obtenidos fueron: aprendizaje basado en problemas 0.818 con 20 elementos, la dimensión 1: Tareas de comunicación 0.774 con 13 elementos y la dimensión tareas de apoyo lingüístico 0.429 con 7 elementos. El segundo cuestionario empleado fue la Escala de Likert para recoger información sobre la variable competencia instrumental traductora, conformada por 26 ítems redactados en forma de afirmaciones. Su contenido fue validado por un grupo de especialistas en el idioma inglés; la fiabilidad fue de 0,948 con 26 elementos para la 
variable competencia instrumental traductora; para la dimensión uso de fuentes de documentación 0.821 con 8 elementos. La dimensión 2, búsqueda de terminología, obtuvo 0.795 con 4 elementos; y la dimensión manejo de aplicaciones informáticas obtuvo 0.938 con 14 elementos. Todos estos resultados se obtienen de los datos de la prueba piloto.

Finalmente, el procedimiento investigativo estuvo centrado en primer lugar en la fundamentación teórico-practica de las variables de estudio, y en el tratamiento de datos se pasó por varias etapas para la fiabilidad por el Alpha de Cronbach; para la prueba de normalidad se aplicó la prueba Kolgomorov-Smirnov, ya que la muestra de datos era mayor que 30. Los resultados aportaron la decisión estadística para la contratación de las hipótesis que fue Rho Spearman, estadístico no paramétrico. A partir de los resultados de la investigación se elaboró la discusión de resultados, conclusiones y recomendaciones. El trabajo investigativo consideró criterios académicos amparados en el manual de estilo APA que aseguran el respeto de autoría en su correcto citado de fuentes y citas bibliográficas.

\section{Resultados}

\section{Tabla 1}

Aprendizaje basado en problemas

\begin{tabular}{lrrr}
\hline & Frecuencia & Porcentaje & Porcentaje acumulado \\
\hline Bajo & 47 & 32,4 & 32,4 \\
Medio & 67 & 46,2 & 78,6 \\
Alto & 31 & 21,4 & 100,0 \\
Total & 145 & 100,0 & \\
\hline
\end{tabular}

Fuente propia del autor 


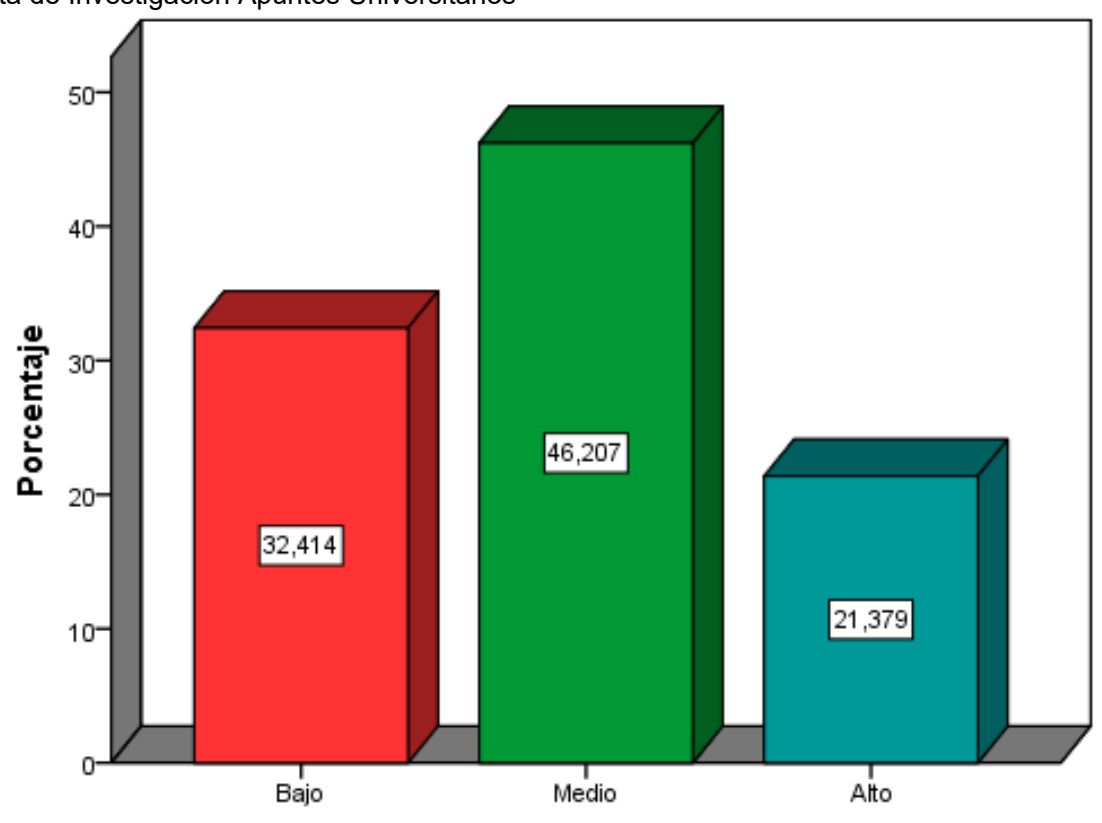

Figura 1. Aprendizaje basado en problemas. Fuente propia del autor

Se puede observar que, en el Aprendizaje basado en problemas, del total (145 participantes), 67 (46,20\%) se ubican en un nivel medio; 47 (32,41\%), en el nivel bajo; y $31(21,37 \%)$ al nivel alto.

Tabla 22

Niveles de Competencia instrumental traductora

\begin{tabular}{lccc}
\hline Niveles & Frecuencia & Porcentaje & Porcentaje acumulado \\
\hline Bajo & 0 & 0,0 & 0,0 \\
Medio & 88 & 60,7 & 60,7 \\
Alto & 57 & 39,3 & 100,0 \\
Total & 145 & 100,0 & \\
\hline
\end{tabular}

Fuente propia del autor 


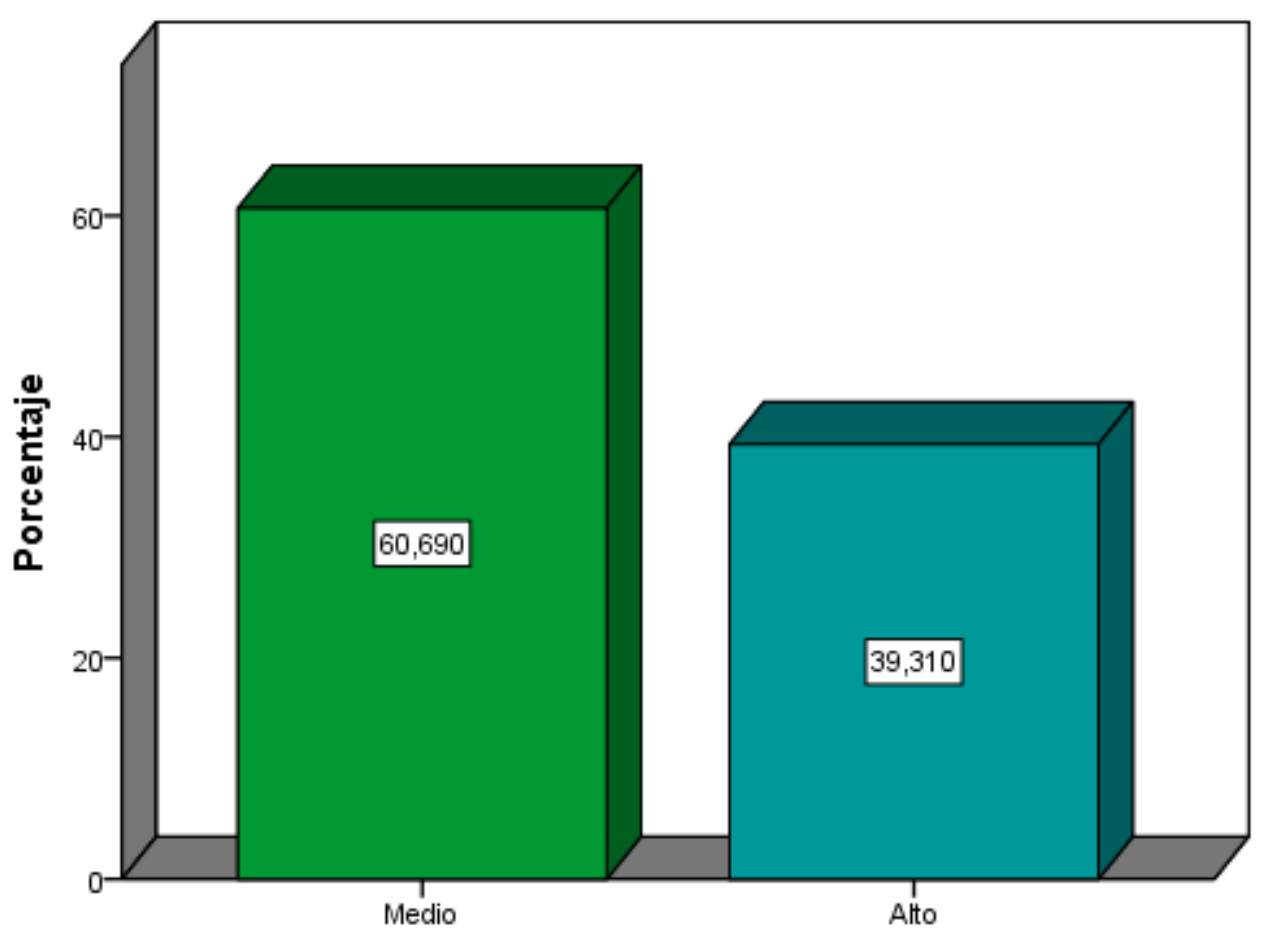

Figura 2. Competencia instrumental traductora

En la Figura 2 se demuestra que, en Competencia instrumental traductora, del total (145 participantes), 88 (60,69\%) se encuentran en nivel medio; $57(39,31 \%)$, al nivel alto; y ningún estudiante corresponde al nivel bajo.

\section{Tabla 33}

\section{Aprendizaje basado en problemas y competencia instrumental traductora}

\begin{tabular}{|c|c|c|c|c|c|c|c|c|}
\hline \multirow{3}{*}{$\begin{array}{l}\text { Aprendizaje } \\
\text { basado en } \\
\text { problemas }\end{array}$} & \multicolumn{6}{|c|}{ Competencia Instrumental Traductora } & \multirow{2}{*}{\multicolumn{2}{|c|}{ Total }} \\
\hline & \multicolumn{2}{|c|}{ Bajo } & \multicolumn{2}{|c|}{ Medio } & \multicolumn{2}{|c|}{ Alto } & & \\
\hline & $\mathrm{n}$ & $\%$ & $\mathrm{n}$ & $\%$ & $\mathrm{n}$ & $\%$ & $\mathrm{~N}$ & $\%$ \\
\hline Bajo & 0 & $0 \%$ & 26 & $18 \%$ & 21 & $14 \%$ & 47 & $32 \%$ \\
\hline Medio & 0 & $0 \%$ & 47 & $32 \%$ & 20 & $14 \%$ & 67 & $46 \%$ \\
\hline Alto & 0 & $0 \%$ & 15 & $10 \%$ & 16 & $11 \%$ & 31 & $21 \%$ \\
\hline Total & 0 & $0 \%$ & 88 & $61 \%$ & 57 & $39 \%$ & 145 & $100 \%$ \\
\hline
\end{tabular}




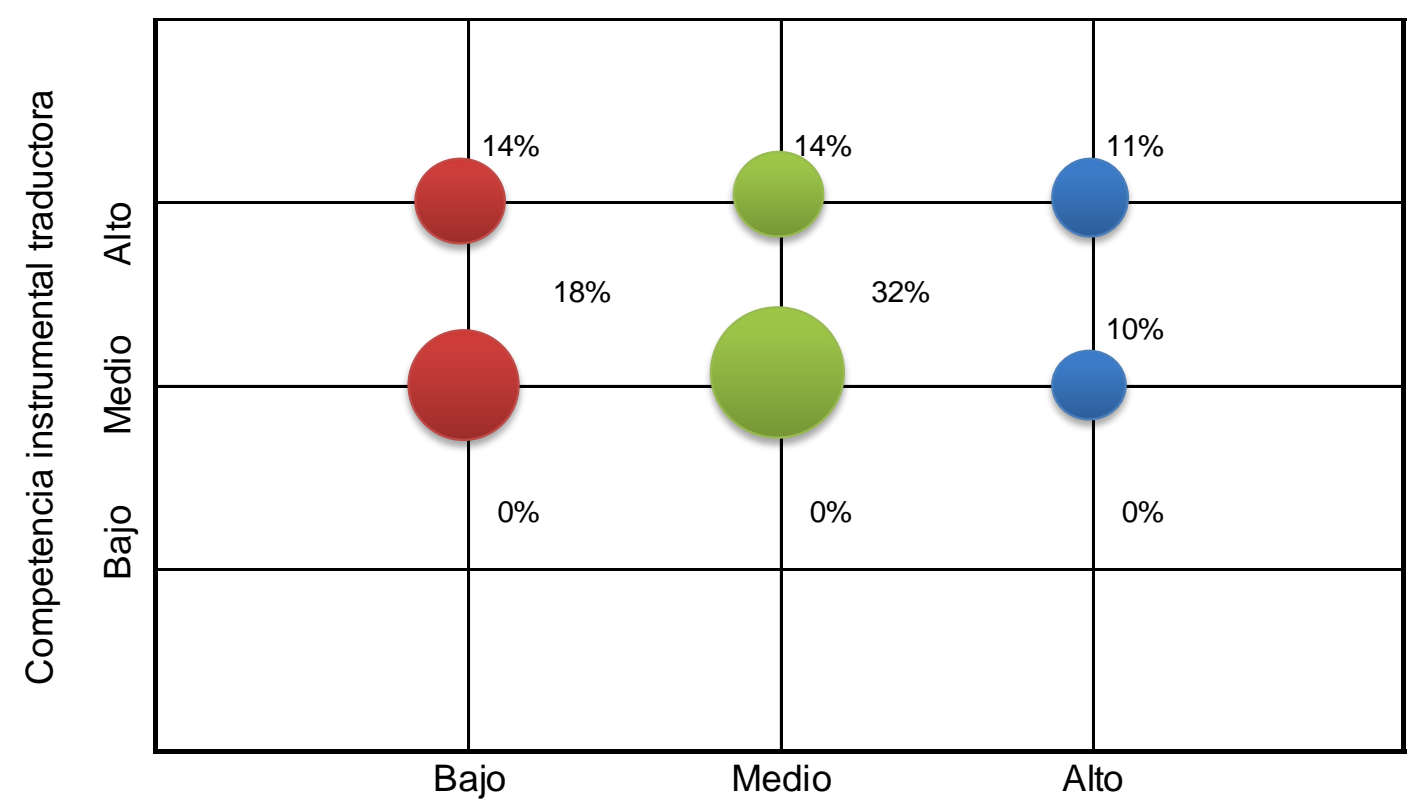

Figura 3. Aprendizaje basado en problemas y competencia instrumental traductora

La tabla 3 y la figura 3 muestran que el $18 \%$ de los participantes se ubica en nivel bajo de Aprendizaje basado en problemas y en un nivel medio en Competencia instrumental traductora; mientras que un $11 \%$ se posiciona en nivel alto en Aprendizaje basado en problemas y al mismo tiempo en un nivel alto de Competencia instrumental traductora.

\section{Tabla 4}

\section{Correlación del aprendizaje basado en problemas y la competencia instrumental} traductora

\begin{tabular}{lllcc}
\hline & & & $\begin{array}{c}\text { Aprendizaje } \\
\text { basado en } \\
\text { problemas }\end{array}$ & $\begin{array}{c}\text { Competencia } \\
\text { instrumental } \\
\text { traductora }\end{array}$ \\
\hline $\begin{array}{l}\text { Rho de } \\
\text { Spearman }\end{array}$ & $\begin{array}{l}\text { Aprendizaje } \\
\text { basado en }\end{array}$ & Coeficiente de correlación & 1,000 &, 016 \\
& problemas & $\mathrm{N}$ &. &, 852 \\
& $\begin{array}{l}\text { Competencia } \\
\text { instrumental }\end{array}$ & Coeficiente de correlación & 145 & 145 \\
& Sig. (bilateral) &, 016 & 1,000 \\
& traductora & $\mathrm{N}$ &, 852 &. \\
\hline
\end{tabular}

Al observar la significancia $\mathrm{p}=0,852$ mayor que $\alpha=0,05$, se rechaza la hipótesis alterna, y se acepta la hipótesis nula. Es decir, el aprendizaje basado en problemas no se correlaciona con la competencia instrumental traductora de manera 
significativa.

Tabla 5

Correlación del aprendizaje basado en problemas y el uso de fuentes de documentación

\begin{tabular}{|c|c|c|c|c|}
\hline & & & $\begin{array}{l}\text { Aprendizaje } \\
\text { basado en } \\
\text { problemas }\end{array}$ & $\begin{array}{l}\text { Uso de fuentes de } \\
\text { documentación }\end{array}$ \\
\hline \multirow{6}{*}{$\begin{array}{l}\text { Rho de } \\
\text { Spearman }\end{array}$} & \multirow{3}{*}{$\begin{array}{l}\text { Aprendizaje } \\
\text { basado en } \\
\text { problemas }\end{array}$} & Coeficiente de correlación & 1,000 &, 065 \\
\hline & & Sig. (bilateral) & - &, 436 \\
\hline & & $\mathrm{n}$ & 145 & 145 \\
\hline & \multirow{3}{*}{$\begin{array}{l}\text { Uso de fuentes } \\
\text { de } \\
\text { documentación }\end{array}$} & Coeficiente de correlación &, 065 & 1,000 \\
\hline & & Sig. (bilateral) & 436 & \\
\hline & & $\mathrm{n}$ & 145 & 145 \\
\hline
\end{tabular}

Como el valor de significancia es $p=0,436$, mayor que $\alpha=0,05$, se rechaza la hipótesis alterna, y se acepta la hipótesis nula. En consecuencia, no existe relación significativa entre el aprendizaje basado en problemas y el uso de fuentes de documentación.

\section{Tabla 6}

Correlación del aprendizaje basado en problemas y la búsqueda de terminología

\begin{tabular}{|c|c|c|c|c|}
\hline & & & $\begin{array}{c}\text { Aprendizaje } \\
\text { basado en } \\
\text { problemas }\end{array}$ & $\begin{array}{l}\text { Búsqueda de } \\
\text { terminología }\end{array}$ \\
\hline \multirow{6}{*}{$\begin{array}{l}\text { Rho de } \\
\text { Spearman }\end{array}$} & \multirow{3}{*}{$\begin{array}{l}\text { Aprendizaje } \\
\text { basado en } \\
\text { problemas }\end{array}$} & Coeficiente de correlación & 1,000 &,- 079 \\
\hline & & Sig. (bilateral) & . & 347 \\
\hline & & $\mathrm{N}$ & 145 & 145 \\
\hline & \multirow{3}{*}{$\begin{array}{l}\text { Búsqueda de } \\
\text { terminología }\end{array}$} & Coeficiente de correlación &,- 079 & 1,000 \\
\hline & & Sig. (bilateral) & 347 & . \\
\hline & & $\mathrm{N}$ & 145 & 145 \\
\hline
\end{tabular}

Al ser la significancia $p=0,347$, mayor que $\alpha=0,05$, se rechaza la hipótesis alterna, y se acepta la hipótesis nula. Por tanto, no se presenta correlación significativa entre el aprendizaje basado en problemas y la búsqueda de terminología. 


\section{Tabla 7}

Correlación del aprendizaje basado en problemas y el manejo de aplicaciones informáticas

\begin{tabular}{|c|c|c|c|c|}
\hline & & & $\begin{array}{l}\text { Aprendizaje } \\
\text { basado en } \\
\text { problemas }\end{array}$ & $\begin{array}{l}\text { Búsqueda de } \\
\text { terminología }\end{array}$ \\
\hline \multirow{6}{*}{$\begin{array}{l}\text { Rho de } \\
\text { Spearman }\end{array}$} & \multirow{3}{*}{$\begin{array}{l}\text { Aprendizaje } \\
\text { basado en } \\
\text { problemas }\end{array}$} & Coeficiente de correlación & 1,000 & -079 \\
\hline & & Sig. (bilateral) & & 347 \\
\hline & & $\mathrm{N}$ & 145 & 145 \\
\hline & \multirow{3}{*}{$\begin{array}{l}\text { Búsqueda de } \\
\text { terminología }\end{array}$} & Coeficiente de correlación &,- 079 & 1,000 \\
\hline & & Sig. (bilateral) & 347 & \\
\hline & & $\mathrm{N}$ & 145 & 145 \\
\hline
\end{tabular}

Al ser el valor de significación $\mathrm{p}=0,079$ mayor que $\alpha=0,05$, se rechaza la hipótesis alterna, y se acepta la hipótesis nula. Esto determina que no se presente correlación significativa entre el aprendizaje basado en problemas y el manejo de aplicaciones informáticas.

\section{Discusión}

Con respecto a la hipótesis general de investigación, no se encontró relación significativa entre el aprendizaje basado en problemas y la competencia instrumental traductora, ya que el valor de significancia fue de $\mathrm{p}=.852$, un valor mayor que el punto estadístico de margen de error: $\alpha=.05$. Por este motivo, se aceptó la hipótesis nula. Se colige de esto que, si ambas variables_además de ser independientes — no muestran evidencia de mutua correlación, sería importante trabajarlas de manera separada o unida, pero sin esperar que se afecten la una a la otra. Esta situación es probable debido a los problemas o tareas propuestas que no han conseguido una significatividad en los estudiantes al ejercer la instrumentalización de su competencia traductora. Este resultado concuerda con lo hallado por Mizab y Bahloul (2016) en su investigación The Integration of Professional Translators' 21st Century Profile in Teaching Translation at Batna University, en Argelia, donde los autores concluyen que en las clases se hace más relevancia a la competencia lingüística que a otras, entre ellas la instrumental. Por ello, trabajos investigativos como los de Escarrá (2013): Characterization of Instrumental Sub-Competence in Cuban Medical Translators based on a New Model of this Competence, establecen la propuesta de una modelización integradora para desarrollar las mencionadas competencias de carácter instrumental en el ejercicio de la traducción e interpretación. Asimismo, se recomienda que los docentes responsables del 
currículo de la carrera realicen constante promoción de las competencias instrumentales en la traducción. Asimismo, Galán (2009) en su estudio La enseñanza de la traducción en la modalidad semipresencial, destaca lo importante de la didáctica en la utilización de materiales de traducción, ya que se apela a niveles profundos de comprensión, identificación, análisis, aplicación y evaluación. Sobre el modo de evaluar los contenidos, se reflexiona que este debe ser un ejercicio de transparencia, donde se establezcan criterios claros de baremación, al establecer rangos en los niveles de calificaciones. Todo lo contrario, encuentra la investigación de Wong (2014), quien en su estudio Sistema de evaluación y el desarrollo de competencias genéricas en estudiantes universitarios, halló mejoría en competencias cognitiva, procedimental y actitudinal; con la aplicación anticipada de una didáctica efectiva, basada en estudios de caso, escalas de estimación, portafolios y rúbricas para evaluar y su correspondiente socialización de las expectativas estudiantiles.

Con referencia a la hipótesis específica primera, se determinó que tampoco el uso de fuentes de documentación se relacionaba de manera significativa con las estrategias basada en tareas, ya que el valor de significancia fue de $p=0,436$, mayor que $\alpha=0,05$. Por consiguiente, se rechazó la hipótesis de trabajo y se aceptó la nula. Esto implica expresamente que, si los participantes en el devenir de sus tareas de traducción lo realizan para cumplir con las exigencias del curso, el estudiante no parte del análisis del documento, no identifica las necesidades y menos revisa los criterios de evaluación que se toman en cuenta; entonces ambas variables, además de ser independientes, responden a naturalezas que no se involucran ni se influyen mutuamente. Esto concuerda con lo comprobado por Massey y Ehrensberger-Dow (2011) quienes en su estudio Technical and Instrumental Competence in the Translator's Workplace: Using Process Research to Identify Educational and Ergonomic Needs, resaltan lo importante del desarrollo de las competencias traductoras instrumentales por sobre las competencias linguiísticas, que, por lo general, se acostumbra a valorar mejor. Este hallazgo coincide con Garay (2015) quien en su trabajo Estilos de aprendizaje e inteligencias múltiples en estudiantes universitarios. Lima. 2014, llega a una similar conclusión de ambas variables, donde solo halla correlación entre el estilo de aprendizaje y la competencia corporal kinestésica con una significancia de $.022(\mathrm{p}<0,05)$. Con esto se comprobó que, de acuerdo a las estrategias que aplique cada participante para lograr el aprendizaje, no presenta relación 
significativa con el empleo de una específica inteligencia traductora hacia una actividad determinada.

Sobre la segunda hipótesis específica, se determinó que no había correlación significativa entre el aprendizaje basado en problemas y la búsqueda de terminología, ya que el valor de significancia fue de $\mathrm{p}=.347$, mayor que $\alpha=0,05$; por tanto, se rechazó la hipótesis de trabajo y se aceptó la nula. Esto determina que los participantes que tienen hábitos en hacer tareas utilizando diversas estrategias no necesariamente buscan la terminología apropiada, ya que la información tratada no corresponde directamente a dicho contenido o quizá no hayan accedido de la manera más idónea a la tarea de buscar terminología. Esto podría explicarse por la ausencia de estas actividades en las sesiones de clase. Todo ello concuerda con Torres (2015) quien en su estudio Desarrollo de la sub-competencia instrumental en estudiantes de traducción, a partir de la enseñanza de recursos terminográficos online, concluye que en las clases se puede observar a algunos estudiantes de traducción que no son tan reflexivos con las particularidades de su perfil profesional y la enorme ventaja que podría representar un empleo más efectivo de las TIC en su ejercicio profesional. Asimismo, se pudo comprobar que los participantes no discernían muy bien entre una problemática y un requerimiento difícil al momento de traducir; se observó también que los participantes suelen hacer permanentes interrupciones durante la traducción del texto origen; tampoco presentaron diligencia en la búsqueda de información en el internet, demostrando con esto que aún no contaban con una efectiva selección de términos confiables. En el mismo sentido, Massey y Ehrensberger-Dow (2011) en su investigación Technical and Instrumental Competence in the Translator's Workplace: Using Process Research to Identify Educational and Ergonomic Needs, remarca lo importante del desarrollo integral en la profesión de traducción, colocando la competencia traductora instrumental sobre la competencia lingüística, que por lo usual es la más predominante. Esta conclusión es complementada con lo encontrado por Lambraño (2014) en Programa Hablar Más y el mejoramiento de la habilidad para hablar Inglés de los estudiantes de Comunicación III de la Licenciatura en Inglés de la Universidad de Córdoba, Colombia, quien llega a la conclusión de que su programa logra mejorar la expresión de ideas en el idioma inglés. Esta investigación comenzó con una media de 1.183 antes de la aplicación de la experiencia del programa y después dicha media subió notablemente a 7.026. Lo mismo pasó en la fluidez, se partió con una media de 2.574 y se llegó luego del programa a 6.122; y con relación a la mejoría de la interacción oral de este idioma, se presentó 
también mejorías, pasando de un nivel inicial de 1.357 en la media a 5.496 después de la aplicación del programa.

Con relación a la tercera hipótesis específica, se comprobó que no existía correlación significativa entre el aprendizaje basado en problemas y el manejo de aplicaciones informáticas, ya que el valor de significancia fue de $\mathrm{p}=.079$, una magnitud mayor a $\alpha=0,05$. Por consiguiente, se rechazó la hipótesis de trabajo y se aceptó la nula. Este hallazgo se puede interpretar como la evidencia de una gran separación entre las actividades de traducción basadas en problemas con su vinculación trunca, parcial o deficiente con las TIC relacionadas a facilitar las tareas de traducción. Asimismo, se puede afirmar que los estudiantes conocen los tipos de tarea de comunicación y de apoyo lingüístico; sin embargo, esto no determina que el estudiante use de manera eficaz las herramientas, la traducción, los formatos de soporte y el montaje que son componentes del manejo de aplicaciones informáticos. Esto es confirmado con los hallazgos de Wang (2016) quien en su estudio Empirical Study on the Computer-aided College English Translation Teaching, llega a la conclusión de que es viable lograr los aprendizajes de un idioma extranjero a través del buen empleo de computadoras. En la misma consonancia, Vega (2012) en su trabajo El método de proyectos y su efecto en el aprendizaje del curso Estadística General en los estudiantes de pregrado, concluye que los logros de aprendizaje mejoran con el empleo de las TIC con un índice de 11 a 14 por más del $50 \%$ de participantes. Comprobando la relación de los métodos de proyectos con los aprendizajes. Esto es complementario con lo hallado por Blumen, Rivero y Guerrero (2011), quienes en Universitarios en educación a distancia: estilos de aprendizaje y rendimiento académico, logran encontrar correlación entre sus variables de trabajo con un $\mathrm{p}<.05$ y asimilación con un $\mathrm{p}<.05$ ), al tiempo que los participantes de posgrado manifestaron una relación más bien moderada en lo referido a la asimilación con un $\mathrm{p}<.05$. Con ello, se consigue el fortalecimiento de los principales resultados de este estudio, aunque todavía resta mucho por desarrollar y fortalecer en la intensa tarea de enseñar y aprender lenguas diversas. Este es el objetivo clave de la carrera de traducción e interpretación, en esencia lo referido a la competencia instrumental traductora, y elemento clave de la presente investigación.

\section{Referencias}

Balcarcel, G. (2006). La corrección del error en clases de inglés como lengua extranjera. Letras, 48(73), 349-363. 
Blumen, S., Rivero, C., y Guerrero, D. (2011). Universitarios en educación a distancia: estilos de aprendizaje y rendimiento académico. Revista de Psicología, 29(2), 225-243.

Boehm, S. (2013). Enseñar a traducir: Un reto para la enseñanza de idiomas

Cerdas-Ramírez, G. y Ramírez-Acosta, J. (2015). La enseñanza de lenguas extranjeras: historia, teoría y práctica. Revista de Lenguas Modernas, 22, 297-316.

Escarrá, A. (2013). Characterization of instrumental sub-competence in cuban medical translators based on a new model of this competence. (Tesis de Maestría). Universidad Central Marta Abreu de las Villas, Santa Clara, Cuba.

Estaire, S. (2011). Principios básicos y aplicación del aprendizaje mediante tareas. MarcoELE, Revista de Didáctica Español Lengua Extranjera, 12, 1-19.

Galán, A. (2009). La enseñanza de la traducción en la modalidad semipresencial. (Tesis doctoral). Universidad Autónoma de Barcelona, Barcelona, España. $\begin{array}{llllll}\text { Recuperado el } & 1 & \text { junio de } & 2017\end{array}$ http://www.tdx.cat/bitstream/handle/10803/5275/aha1de2.pdf

Garay, L. E. (2015). Estilos de aprendizaje e inteligencias múltiples en estudiantes universitarios. (Tesis doctoral). Universidad San Martín de Porres, Lima, Perú.

Jerez-Naranjo, Y., y Garófalo-Hernández, A. (2012). Aprendizaje basado en tareas aplicado a la enseñanza de las telecomunicaciones. Ingeniería Electrónica, Automática y Comunicaciones, 33(3), 1-7. Recuperado el 24 de marzo de 2017 desde http://scielo.sld.cu/pdf/eac/v33n3/eac01312.pdf

Kelly, D. A. (2002). Un modelo de competencia traductora: bases para el diseño curricular. Puentes, 1, 7-20. Recuperado el 3 de marzo de 2017 desde http://wdb.ugr.es/ greti/revista-puentes/pub1/02-Kelly.pdf

Lambraño, E. C. (2014). Programa Hablar Más y el mejoramiento de la habilidad para hablar inglés de los estudiantes de comunicación III de la Licenciatura en Inglés de la Universidad de Córdoba, Colombia. (Tesis de maestría). Universidad Peruana Unión, Lima, Perú. Recuperado el 15 mayo de 2017 desde http://repositorio.upeu.edu.pe/handle/UPEU/276

Lobato, J. (2011). Propuesta didáctica para las clases de traducción especializada: el aprendizaje basado en proyectos. http://www.tonosdigital.es/ojs/index.php/tonos/article/viewFile/991/639

Marangon, G. (2012) Consideraciones en torno a los métodos de enseñanza de lenguas extrajeras y a las teorías de aprendizaje. Filología y Lingüística, 38(2), 121-134. 
Massey, G. y Enhrensberger-Dow, M. (2011). Technical and instrumental competence in the translator's workplace: using process research to identify educational and ergonomic needs. Revista ILCEA, Revue de I'Institut des langues et cultures d'Europe, Amérique, Afrique, Asie et Australie, 14, 1-15. Recuperado el 2 de marzo de 2017 desde https://ilcea.revues.org/1060?lang=es

Mizab, M. y Bahloul, A. (2016) The integration of professional translators' 21st Century Profile in Teaching Translation at Batna University, Arab World English Journal, 3, 187-209.

Novoa Castillo, P. (2012) Uso de los mapas mentales como instrumento de enseñanza en la comprensión lectora de textos narrativos en los alumnos del I ciclo de la Universidad de Ciencias y Humanidades (Tesis de maestría). Universidad Nacional de Educación Enrique Guzmán y Valle, Lima, Perú.

Novoa-Castillo, P. (2019) El mapa mental armónico en la comprensión y producción de textos narrativos en estudiantes de pregrado de la UCV (Tesis de doctorado), Universidad César Vallejo, Lima, Perú.

Novoa-Castillo, P., Cancino-Verde, F., Flores-Sotelo, W., y Nieto-Gamboa, J. (2018). El mapa mental armónico en la comprensión de textos narrativos en estudiantes universitarios. Propósitos y Representaciones, 6(2), 541-573. En http://dx.doi.org/10.20511/pyr2018.v6n2.243.

Novoa-Castillo, P., Cancino-Verde, F., Flores-Sotelo, W., y Nieto-Gamboa, J. (2018). The harmonic mind map in the comprehension of narrative texts in university students. Journal of Educational Psychology-Propositos y Representaciones, 6(2), 575-606.

Perkins, G. (2015) Metodología de la traducción directa del inglés al español. Materiales didácticos para traducción general y especializada. Reseñas, 19(2), 298-329.

Recino--Pineda, U., y Laufer, M. (2013). Aprendizaje basado en tareas en la enseñanza comunicativa de lenguas extranjeras. Edumecentro, 2(3), 20-27.

Silva, D. (2017) Aprendizaje basado en tareas y competencia instrumental traductora en estudiantes de una universidad privada, Lima. (Tesis de maestría) Universidad César Vallejo, Lima, Perú. 
Torres, J. J. (2015). Desarrollo de la sub-competencia instrumental en estudiantes de traducción, a partir de la enseñanza de recursos terminográficos online (Tesis de maestría). Universidad Autónoma de Manizales, Manizales, Colombia.

Vega, E. (2012). El método de proyectos y su efecto en el aprendizaje del curso Estadística General en los estudiantes de pregrado. Revista Digital de Investigación en Docencia Universitaria, 6(1). https://doi.org/10.19083/ridu.6.40.

Wang, B. (2016). Empirical study on the computer-aided college english translation teaching. International Journal of Emerging Technologies in Learning, 11(12), 68-71.

Wong, E. M. (2014). Sistema de evaluación y el desarrollo de competencias genéricas en estudiantes universitarios. (Tesis doctoral). Universidad de San Martín de Porres, Lima, Perú. 